\title{
Formulations of Classical Mechanics
}

\author{
Jill North \\ (Draft of March 20I9; final version forthcoming in A Companion to the Philosophy of \\ Physics, Eleanor Knox and Alastair Wilson, eds., Routledge)
}

\begin{abstract}
I outline three formulations of classical mechanics, Newtonian, Lagrangian, and Hamiltonian mechanics, that are ordinarily seen as fully equivalent-notational variants of a single theory. I point to various differences, both mathematical and metaphysical, that may be significant enough to warrant their being distinct theories.
\end{abstract}

\section{Introduction}

Classical mechanics is the physical theory with which we are most familiar, the one we first encounter in school. Philosophers tend to regard classical mechanics as metaphysically unproblematic. At first glance, it does appear straightforward: the theory is fundamentally about particles, with intrinsic features like mass, ${ }^{\mathrm{I}}$ that move around in three-dimensional space in response to various forces, which arise via interactions between the particles. It seems as though, if any physical theory is metaphysically perspicuous, classical mechanics is. But the theory is not as clear-cut as it initially seems. Our familiarity misleads us.

The reason is not just that classical mechanics ultimately runs into the kind of trouble that presaged quantum mechanics. Even taking it to be the true fundamental theory of a world,$^{2}$ classical mechanics does not offer as candid a picture of things as we tend to think. One reason for this is that there are different formulations, which are generally claimed to be equivalent by physics books, but which are at least not obviously equivalent-neither in terms of the mathematical structure they use, nor in terms of the physical world they describe.

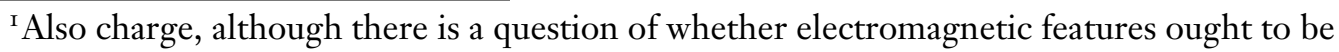
considered part of classical mechanics (see for instance note 9).

${ }^{2}$ Of course, because of the previously-mentioned troubles, it is not clear that classical mechanics can be a true fundamental theory of a world, but set that aside here.
} 
What I want to do in this chapter is to outline the three leading formulations of classical mechanics, and to raise some questions about them, the chief one being: Are these genuinely equivalent formulations, as usually thought? If so, in what sense are they equivalent? If not, in what way(s) do they differ? Another way to put the focal question of this chapter is by means of a title of Mark Wilson's (20 I3): "What is 'Classical Mechanics', Anyway?” Indeed, since the terms 'classical mechanics' and 'Newtonian mechanics' "are used virtually synonymously" (Spivak, 2010, 7), one aim of this chapter is to suggest that it is not right to do so. There are different versions of classical mechanics, which might even amount to distinct theories. A related aim is to show that there are interesting philosophical questions that arise in the context of classical mechanics. Classical mechanics merits the attention of philosophers, who often disregard it as either too perspicuous or too outdated to warrant much discussion. ${ }^{3}$

Although this chapter is limited to classical mechanics, it should be clear that a host of general questions in the philosophy of physics and science are touched upon, such as: What is the right notion of theoretical equivalence-when are two scientific theories mere notational variants? How do we interpret a scientific theory-how do we figure out the nature of the world according to a theory? When faced with different theories or formulations, how do we choose which one to adopt? Must we choose?

\section{Three formulations}

I will outline the three main formulations of classical mechanics-Newtonian, Lagrangian, and Hamiltonian mechanics-in relatively standard ways, before turning to some questions about them. ${ }^{4}$ My focus will be on the dynamical laws, since this is where much of the action lies in comparing and contrasting the different formulations.

\section{I. Newtonian mechanics}

Newtonian mechanics might be the only formulation one comes across, the others typically not introduced until more advanced college courses. In the Newtonian mechanics of point-particles-point-sized physical objects with intrinsic features such as mass ${ }^{5}$ - two sets of coordinates specify a system's fundamental

\footnotetext{
${ }^{3}$ A recent book-length exception: Sklar (2013).

4There are other varieties I don't discuss, such as in terms of Poisson brackets, HamiltonJacobi theory, or four-dimensional spacetime geometry.

5This is the fundamental ontology assumed here. Wilson (2013) discusses the classical mechanics of rigid bodies and continua, and complications involved in trying to encompass all
} 
state at a time: the positions and velocities, or momenta, of all the particles. Assuming the particles are free to move around in three-dimensional physical space, these coordinates will each have three components, one along each spatial dimension.

For a system containing $n$ particles, the total state is specified by $6 n$ coordinates: three coordinates for the position and three coordinates for the velocity for each particle in the system. It turns out to be extremely useful to represent all the possible states of a system in a mathematical space called the statespace, each point of which represents a different possible fundamental state of the system. Since we need $6 n$ coordinates to specify the state of a system, the statespace will have $6 n$ dimensions.

Different curves through the statespace represent different possible histories of the system, different sequences of its fundamental states over time. (The curves are parameterized by time. ${ }^{6}$ ) These histories are given by the dynamical law(s), in this case Newton's second law: ${ }^{7}$

$$
\Sigma \mathbf{F}_{i}=m_{i} \mathbf{a}_{i}=m_{i} \ddot{\mathbf{x}}_{i}
$$

$\Sigma \mathbf{F}_{i}$ indicates the sum of the forces-which are vector quantities, written in bold-on a given particle labeled by $i$ ( $i$ ranges from $\mathrm{I}$ to $n$, for $n$ particles in the system); $m_{i}$ is the particle's mass; $\mathbf{a}_{i}$, or $\ddot{\mathbf{x}}_{i}$, is the particle's acceleration, the second derivative of its position with respect to time, also vector quantities. (A dot over a quantity indicates a derivative with respect to time of that quantity.) In other words: $\sum_{j \neq i} \mathbf{F}_{i j}=m_{i} \mathbf{a}_{i}$, where $\sum_{j \neq i} \mathbf{F}_{i j}$ is the sum of the forces on the given particle due to all the other particles (both in the system and external to it).

The above is a vector equation. There is one such equation for each particle in each component direction-three equations per particle in three-dimensional space. These equations can be grouped together into one master equation, which says how the point representing the state of the entire system moves through the statespace over time. Given the initial state of a system and the total forces acting on it, integrating (twice) yields a unique solution, or history: the laws are deterministic. ${ }^{8}$ A solution picks out a trajectory in the statespace, which represents the paths of all the particles through ordinary space.

of these within a single theory. See Hall (2007, 5.2); Allori (2017); Esfeld et al. (2018) on the non-standard idea that particles don't have fundamental intrinsic properties.

${ }^{6}$ Alternatively, time can be included as an additional dimension of the statespace.

7 Another familiar version of the law, ordinarily seen as equivalent to the above, is in terms of momentum: $\Sigma F=\dot{p}$. See Hicks and Schaffer (20I7) on whether these are genuinely equivalent.

${ }^{8}$ Whether the theory really is deterministic is an interesting question. Apparent counterexamples are in Earman (1986) and Norton (2008); see also Malament (2008) and Wilson (2009). 
Equation $\mathrm{I}$ is the fundamental dynamical equation of the theory. Newton's second law predicts the motion of every particle, in any situation. What forces there are will depend on the types of particles involved, and to calculate the forces we will need additional rules, like the law of gravitation. But this one dynamical law predicts any system's behavior, once given those forces.

Two other laws of Newtonian mechanics as standardly presented are important to the theory as a whole, but will play a less central role here. Newton's first law says that an object continues with uniform velocity unless acted on by a net external force. This law helps define what it is for an object to not accelerate, or to travel inertially (with the second law saying what happens when an object is subject to a net force that yields an acceleration). Newton's third law tells us about the nature of forces. It is often stated in "action-reaction" form: to every action there is an equal and opposite reaction; when one object exerts a force on a second object, the second simultaneously exerts a force equal in magnitude and opposite in direction on the first. This law tells us that forces come in pairs, as the result of interactions between two objects. It "describes the forces to some extent" (Feynman et al., 2006, 9.I), with the particular force laws telling us that forces don't depend on anything other than the types of particles and their spatial separations, and that they are central forces, directed along the line between the particles. (Conservative forces, derivable from a potential.) ${ }^{9}$

\subsection{Lagrangian mechanics}

In Lagrangian mechanics, two sets of so-called generalized coordinates describe systems' fundamental states: the generalized positions, $q_{i}$, and their first time derivatives, the generalized velocities, $\dot{q}_{i}$ ( $i$ from I to $n$ for $n$ particles in the system). As in Newtonian mechanics, we need $6 n$ coordinates to completely specify the state of a system of $n$ particles at a time: three generalized position and three generalized velocity coordinates per particle. But unlike in Newtonian mechanics, these don't have to be ordinary position and velocity coordinates.

\footnotetext{
${ }^{9}$ There are questions surrounding the further restrictions that forces be central and conservative. It is usually thought that nonconservative forces, like frictional ones depending on velocity, arise from fundamental conservative ones. As Feynman notably put it, "there are no nonconservative forces!” (2006, I4.4). Newton himself didn't restrict forces this way; Feynman suggests that it is an additional empirical posit (compare Baez: "It is a simplifying assumption that has withstood the test of time and experiment" $(2005,6)$ ). Hutchison (1993) argues that the restriction is illegitimate; Callender (1995) responds. The restrictions are assumed in standard proofs of energy conservation and other theorems. (This is one place the question of electromagnetic features (note I) comes into play. Consider the magnetic force on a moving charge, which doesn't satisfy the restrictions.) Concerns over the above have led a couple of authors to doubt the equivalence of the formulations: Lanczos (I970, $77 \mathrm{nI}$ ); Gallavotti (I983, ch. 3). See also Hertz (I 899); Wilson (2009, 20I3, forthcoming) on reasons to doubt their equivalence.
} 
(They are called generalized positions and velocities by analogy to ordinary positions and velocities.) Generalized coordinates can be any set of independent parameters that completely specify a system's state. Generalized positions can have units of energy, or length squared, or an angle, or can even be dimensionless. We can use any kind of coordinates that are suited to a system, the choice usually dictated by the number of degrees of freedom of the system and the topology of the spatial region in which the particles are free to move around. ${ }^{\text {I0 }}$ For a pendulum, for example, we might use the angle $\theta$ that the cord makes with respect to the vertical as the generalized position, with $\dot{\theta}$ the generalized velocity.

The Lagrangian statespace is a $6 n$-dimensional space with the structure of a tangent bundle. This comprises a $3 n$-dimensional space in which we represent the generalized positions (the configuration space), plus the $3 n$-dimensional tangent space at each point, for the generalized velocities, which are tangent to the generalized positions. Each point in this space picks out a generalized position and generalized velocity for each particle in the system. Standard labels are $Q$ for the configuration space (the base space of the tangent bundle), $T_{q} Q$ for the tangent spaces (the fibers, one for each $q$ in $Q$ ), and $T Q$ for the entire statespace, sometimes called the velocity phase space. Note that the configuration space is what represents the physical space the particles move around in. Given the freedom in generalized coordinates, it needn't do so in an obvious way, yet the structure of physical space will still be coded up in the structure of $Q$.

The dynamical laws, called the Lagrange or Euler-Lagrange equations, say how the point representing a system's state moves through the statespace over time, given a scalar function called the Lagrangian, $L$. At each point in the statespace, this function assigns a number, typically equal to the system's kinetic energy, $T$, minus its potential energy, $V .{ }^{I I}$ Although this gives the Lagrangian as defined on TQ, we can think of it as coding up information about particles' ordinary spatial features, those that are relevant to their energies, so that it is ultimately about goings-on in three-dimensional space. ${ }^{12}$ The motion of an $n$-particle system in three-dimensional space is then given by $n$ second-order equations, ${ }^{13}$ one for each particle in each direction; i.e., one for each degree of

\footnotetext{
${ }^{10}$ The number of degrees of freedom is the number of independent parameters "necessary and sufficient for a unique characterization" of the system (Lanczos, I970, I0). There are some mild constraints on generalized coordinates: José and Saletan (I998, 2.I.2).

${ }^{11}$ Standard examples in which it does not have this form come from outside point-particle mechanics, such as electromagnetism or special relativity: José and Saletan (I998, 2.2.4); Goldstein et al. (2004, 7.9).

${ }^{12}$ More on the different ways of conceiving of quantities like $L$ in section 3.2 .

${ }^{13}$ These can be seen as first-order equations defined on all of TQ: José and Saletan (1998, 2.4).
} 
freedom (three per particle in three-dimensional space):

$$
\frac{d}{d t}\left(\frac{\partial L}{\partial \dot{q}_{i}}\right)-\frac{\partial L}{\partial q_{i}}=0 .
$$

Given $L$, these equations uniquely determine the motion for an initial state characterized by the generalized position and generalized velocity of each particle in the system. A solution, found by integrating, gives a function or trajectory on $Q$, which represents the particles' motions through physical space. (Solutions are curves through $T Q$, which are projected onto $Q$.)

Picture the statespace for a particle moving on a one-dimensional circle: figure $\mathrm{I}$. This is a two-dimensional space, with each point picked out by two

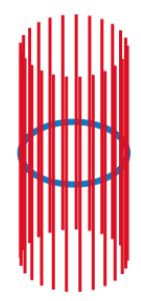

Figure I: Two-dimensional tangent bundle (image from Wikipedia)

coordinates $(q, \dot{q})$. The circle represents the different possible values of the generalized position coordinate, and the lines the different possible values of the generalized velocity. Curves through this space represent different possible histories of the system. The figure could represent the statespace of a point-mass pendulum, for instance, with the circle representing the different values of $\theta$ and the lines the values of $\dot{\theta}$. Keep in mind that this is "just about the only easily visualized nontrivial TQ" (José and Saletan, 1998, 94). With more degrees of freedom, things quickly become difficult to picture.

Briefly note three interesting, interrelated differences between the Lagrangian and Newtonian formulations. ${ }^{14}$ First, Lagrangian mechanics relies on a scalar energy function to determine a system's motion, whereas Newtonian mechanics relies on forces, which are vector quantities. Second, Lagrangian mechanics takes a more "holistic" approach to describing systems' motions, in terms of the energy of the system as a whole. By contrast, the Newtonian formulation "is intrinsically a particle-by-particle description" (Sussman and Wisdom, 2014, 3), given in terms of the forces on each individual particle due to every other particle. Third, Lagrangian mechanics is a more coordinate-independent formulation of

\footnotetext{
${ }^{14}$ Discussion in Lanczos (1970). See Butterfield (2004) on Lagrangian mechanics in particular.
} 
the dynamics, in that we can substitute any kind of coordinates for $q$ and $\dot{q}$ in equation 2. The central equation of Newtonian mechanics, on the other hand, contains an implicit preference for Cartesian coordinates. We can of course use other kinds of coordinates, but the form of the equation will differ; whereas "Lagrange's equations, unlike Newton's, take the same form in any coordinate system" (Taylor, 2005, 237). (The form of an equation is the form as a function of its variables, a standard notion in physics. ${ }^{15}$ )

\subsection{Hamiltonian mechanics}

Hamiltonian mechanics shares a special kinship with Lagrangian mechanics, more so than with Newtonian mechanics. Here too, a scalar energy function determines the motion, and the equations are formulated in terms of generalized coordinates. There are also some notable differences. Hamiltonian mechanics uses a different energy function and a different kind of generalized coordinate, with the result that the dynamical equations and statespace also differ.

The Hamiltonian coordinates are called canonical coordinates. These are the generalized positions, $q_{i}$, and generalized momenta, $p_{i}$. (As before, $i$ ranges from 1 to $n$ for $n$ particles, with three of each coordinate per particle in threedimensional space.) Here the statespace is the cotangent bundle of configuration space, $T^{*} Q$. This comprises the configuration space, $Q$, together with the cotangent space, $T^{*}$ (dual to the tangent space), at each point in $Q$, for the generalized momenta (which are covectors, or one-forms). This is a $6 n$-dimensional manifold, each point of which picks out a generalized position and generalized momentum for each particle in the system. It is often called the momentum phase space, or simply the phase space. ${ }^{16}$

The scalar function that describes a system's motion is called the Hamiltonian, $H$, and is (typically ${ }^{17}$ ) equal to the total energy of the system, that is, the potential energy plus the kinetic energy, instead of the difference between them as in Lagrangian mechanics. The dynamical laws are a set of $2 n$ first-order equations, two for each particle in each direction; two sets of equations for each degree of freedom:

$$
\dot{q}_{i}=\frac{\partial H}{\partial p_{i}}, \quad \dot{p}_{i}=-\frac{\partial H}{\partial q_{i}} .
$$

These equations, called the Hamiltonian or canonical equations, uniquely deter-

\footnotetext{
${ }^{15}$ Brading and Castellani (2007, I343). Newton's equation, in standard form, is a linear function of acceleration or second derivative of position. Contrast the form in polar coordinates: Taylor (2005, eq. I.48).

${ }^{16}$ In fact, a Hamiltonian statespace can have a more general structure than this: North (2009).

${ }^{17}$ See Goldstein et al. (2004); Taylor $(2005,7.8)$ for conditions under which this holds.
} 
mine a system's motion, given an initial state specified by the canonical positions and momenta of each particle in the system.

Hamiltonian and Lagrangian mechanics are both more coordinate-independent formulations than Newtonian mechanics. Both are given in terms of generalized coordinates, with the result that the dynamical equations retain their form regardless of which coordinates we use. ${ }^{8}$ The reason is that the Lagrangian and Hamiltonian functions, which determine the motion, are scalar functions. In Newtonian mechanics, by contrast, vector quantities-forces-determine the motion. Although vectors are coordinate-independent objects, their components change with the coordinate system. (Vectors can be defined by means of how their components transform under coordinate changes.) And as Feynman puts it, "The general statement of Newton's Second Law for each particle...is true specifically for the components of force and momentum [or acceleration] in any given direction" (2006, I0.3), since "any vector equation involves the statement that each of the components is equal" (2006, I I.9). Scalars are even more coordinateindependent than that, being completely unaffected by coordinate changes, not even "altering component-wise." (The form of a scalar function such as $L$ or $H$ may change with the coordinate system, but not the scalar value, nor the form of the equation in which $L$ or $H$ appear.)

\subsection{Example: plane pendulum}

Briefly work through a simple example to get a feel for the different flavor of each formulation. Consider a vertical plane pendulum (figure 2), which moves through two spatial dimensions. (Assume the usual idealizations: a frictionless, rigid cord; point-mass bob; negligible air resistance; uniform gravitational field.) Use each formulation to find the equation that describes the pendulum's position as a function of time. In fact, we will see, each formulation yields the same equation of motion.

To use Newton's law, first choose a rectangular coordinate system. Let $y$ be in the radial direction, directed along the cord, and let $x$ be in the direction tangential to the bob's path. Resolve the forces into their components in this coordinate system. There are two forces on the bob: the tension along the string and the downward gravitational force. The component of the gravitational force in the direction of the bob's acceleration along the path (the tangential force) is $m g \sin \theta$, where $\theta$ is the angle the string makes with respect to the vertical, as shown in the figure.

There are two component equations of Newton's law, one for each direction

\footnotetext{
${ }^{18}$ The equations can be given in coordinate-free, not just coordinate-independent, terms: José and Saletan (I998, eqs. 3.87 and 5.70).
} 


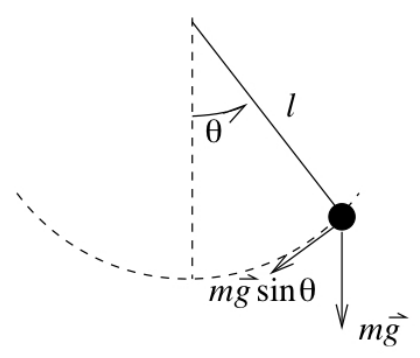

Figure 2: Plane pendulum (MIT OCW)

of our coordinate system: $F_{x}=m a_{x}$ and $F_{y}=m a_{y}$. Plugging in the relevant force components yields $F_{x}=-m g \sin \theta=m a_{x}$ (the negative sign because the gravitational force points downward) and $F_{y}=T-m g \cos \theta=m a_{y}$, with $T$ the tension in the string. But $a_{y}=0$, so we ignore the second equation and this force when solving for the equation of motion. ( $T$ has no component in the direction of nonzero acceleration; it is merely a "constraint force.")

The arclength, $s$, which measures the distance traveled by the bob along the curved path, is given by $s=l \theta$. The second derivative of this quantity, $\ddot{s}=l \ddot{\theta}$, is the acceleration along the path. Plug into the $x$-component equation of Newton's law to get the equation of motion:

$$
-g \sin \theta=l \ddot{\theta} .
$$

We get the same equation of motion using Lagrangian mechanics via a different route. We could use rectangular coordinates, but things are simpler if we use generalized coordinate $\theta$, with $\dot{\theta}$ the generalized velocity; plug these directly into equation 2 to get the solution. In this way, we can effectively treat $\theta$ and $\dot{\theta}$ as ordinary position and velocity coordinates.

First calculate the Lagrangian, $L=T-V$. The kinetic energy $T=\frac{1}{2} m v^{2}=$ $\frac{1}{2} m(l \dot{\theta})^{2}$. (The arclength is $s=l \theta$, the velocity its first time derivative.) The potential energy $V=-m g l \cos \theta$, setting the zero at the height of the pivot point where $\theta=\frac{\pi}{2}$. (Gravitational potential energy $=m g y$, with $y$ the vertical distance from a chosen zero.) This gives us $L=\frac{1}{2} m(l \dot{\theta})^{2}+m g l \cos \theta$. Calculate the following derivatives (here treating $\theta$ and $\dot{\theta}$ as independent variables, even though one is really defined as the time derivative of the other): $\frac{\partial L}{\partial q}=\frac{\partial L}{\partial \theta}=-m g l \sin \theta$ and $\frac{\partial L}{\partial \dot{q}}=\frac{\partial L}{\partial \dot{\theta}}=m l^{2} \dot{\theta}$, so that $\frac{d}{d t}\left(\frac{\partial L}{\partial \dot{\theta}}\right)=m l^{2} \ddot{\theta}$. Finally, plug into equation 2: $m l^{2} \ddot{\theta}-(-m g l \sin \theta)=0$, i.e. $l \ddot{\theta}+g \sin \theta=0$, which is equation 4 . 
In Hamiltonian mechanics, we first find the Hamiltonian, $H=T+V$. Given $L$ calculated above, we can see that $H=\frac{1}{2} m(l \dot{\theta})^{2}-m g l \cos \theta$; but we need to write this in terms of canonical coordinates. To find the generalized momentum $p_{\theta}$, which is "conjugate" to the position variable $\theta$, use this equation: $p=\frac{\partial L}{\partial \dot{q}}$, which is often taken to be the definition of the generalized momentum. ${ }^{19}$ Using the equation $p_{\theta}=\frac{\partial L}{\partial \dot{\theta}}$, we find $p_{\theta}=m l^{2} \dot{\theta}$, so that $\dot{\theta}=\frac{p_{\theta}}{m l^{2}}$, which we can use to eliminate $\dot{\theta}$ from the expression for $H$. Thus, $H=\frac{1}{2} m\left(l \frac{p_{\theta}}{m l^{2}}\right)^{2}-m g l \cos \theta=$ $\frac{p_{\theta}^{2}}{2 m l^{2}}-m g l \cos \theta$. Now we can find the equation of motion for the pendulum using the Hamiltonian equation $\dot{p}=-\frac{\partial H}{\partial q}$; that is, $\dot{p}_{\theta}=-\frac{\partial H}{\partial \theta}=-m g l \sin \theta$. Differentiate $p_{\theta}=m l^{2} \dot{\theta}$ to get $\dot{p}_{\theta}=m l^{2} \ddot{\theta}$, and plug into the equation for $\dot{p}_{\theta}$ to get $m l^{2} \ddot{\theta}=-m g l \sin \theta$; i.e. $l \ddot{\theta}=-g \sin \theta$, which is again equation 4 .

\section{Equivalent formulations?}

We get the same equation of motion for the pendulum regardless of which formulation we use. This turns out to be true in general. It is often simpler to use Lagrangian or Hamiltonian mechanics, since we do not have to calculate the various component forces on each particle. Nonetheless, it is generally agreed that each formulation suffices for describing the motion of any classical mechanical system. The difference seems to be just a matter of calculational convenience.

Indeed, physics books typically state, and go on to prove, an equivalence among the three formulations, by showing that the dynamical equations are interderivable. ${ }^{20}$ A typical route is to begin with Newton's laws, derive the Lagrangian and Hamiltonian equations from them, and then show that the derivation can go the other way. Thus José and Saletan, at the beginning of their chapter on Lagrangian mechanics, following the one on Newtonian mechanics, write, "In this chapter we show how the equations of motion can be rewritten....We should emphasize that the physical content of Lagrange's equations is the same as that of Newton's" $(1998,48)$. They then show that Hamilton's equations, in turn, can be derived from Lagrange's, and vice versa, concluding that these all "contain the same information" (I 998, 207). Another book concludes that,

${ }^{19}$ The above is an instance of a Legendre transformation, which can be used to change back and forth between Hamiltonian and Lagrangian coordinates, energy functions, and statespaces: Lanczos (1970, ch. 6); Arnold (1989, 3.I4); José and Saletan (1998, ch. 5).

${ }^{20}$ See for example Arnold (1989); Marion and Thornton (I995); Hand and Finch (I998); José and Saletan (1998); Talman (2000); Goldstein et al. (2004); Baez (2005); Taylor (2005); see also Feynman (1965, ch. 2). 
"From the point of view of the physicist this division [into the three formulations] is rather artificial.... The segregation is based entirely on the mathematical methods used" (Talman, 2000, I63). It certainly seems like these are simply "alternative statements of the laws" (Marion and Thornton, I995, 2 I3), with "nothing new... added to the physics involved" (Goldstein et al., 2004, 334) when

we pass from one formulation to another. The standard view is that the three formulations are equivalent in all relevant respects: mere notational variants.

I want to urge caution in adopting the standard view, however. The alleged equivalence is not as straightforward as the above statements would have us believe. The reason is that there are some differences among the formulations, and it is not obvious that they are as superficial as usually thought. Draw a rough distinction between two kinds of differences: mathematical and metaphysical. I won't go into these in detail, but will point to places where there is a case to be made that the differences go deeper than usually thought.

\section{I. Mathematical differences}

It is important to keep in mind that two things can be equivalent in some ways while differing in other ways: two objects can share a shape yet have different colors or patterns; two planes can share a distance measure yet differ in whether they have a preferred direction or location. In mathematics more generally, two objects are equivalent when there is the relevant structure-preserving mapping between them; the objects are then equivalent with respect to that structure. All of which is to say that, even if the three formulations of classical mechanics are equivalent in the ways that physics books say, they could still be nonequivalent in other ways. The question is whether they are equivalent, full stop. The answer depends on whether what differences there are matter in any way.

There is one patent mathematical difference among them: the formulations use different symbols, in equations that do not "look" the same. The standard view is that this difference does not matter. Consider the change from Cartesian to polar coordinates to describe a Euclidean plane, or from one set of Cartesian coordinates to another that is rotated or translated with respect to the first. Some things will be different when we switch to the other coordinate system-points get labeled with different numbers, for example-but we know that nothing has really changed. The plane remains the same; we have simply used a different, equally legitimate way of describing it. The standard view is that the differences among the formulations of classical mechanics are like the differences among the coordinate-based descriptions of the plane: just a change in the coordinates or variables being used to describe the very same physics.

Yet there are some reasons for hesitation about this. Take Newtonian me- 
chanics, on the one hand, and Lagrangian and Hamiltonian mechanics, on the other. The latter are comparatively coordinate-independent formulations of classical mechanics. This suggests that they more directly get at the nature of classical mechanical reality, apart from our descriptions of it-just as the metric tensor on a Euclidean plane, rather than any coordinate-dependent distance formula, more directly captures the intrinsic structure of the plane. (The familiar distance formula derived from the pythagorean theorem, for instance, assumes Cartesian coordinates and won't work in other types of coordinates, even though the distance between any two points is the same regardless of coordinates.) This, in turn, suggests that we have reason to prefer these formulations. Physics prizes coordinate-independence, and with good reason. ${ }^{21}$ Since there is freedom in which coordinate system to use, any choice we do make will be to some extent arbitrary-a conventional choice made from among equally good descriptions. (Recall the different coordinate systems for the plane.) We can be misled into thinking that coordinate-dependent features, which rest on an arbitrary choice in description, reflect genuine features of reality. ${ }^{22} \mathrm{~A}$ formulation that is independent of coordinates is then preferable, other things being equal, when it comes to interpreting what physics says about the world. So that even if the equations are all inter-derivable in some sense, there is also a sense in which they are not all mathematically on a par; a sense in which they are not completely equivalent. Some of them may more directly represent physical reality than others.

We can go further. For the way in which the formulations differ in their reliance on coordinates suggests particular physical differences among them. Newtonian mechanics contains an implicit preference for Cartesian coordinates, in which its core equation takes the standard form. A preference for Cartesian coordinates, in turn, is indicative of a Euclidean metric structure. This suggests that the spatial structure of a Newtonian world is Euclidean. (This structure will be reflected in the statespace, in particular the configuration space, which codes up the structure of physical space.) Lagrangian mechanics allows for a wider range of coordinates. As a result, it does not constrain the spatial metric in the same way. This suggests that the physical space of a Lagrangian world (as well as the statespace in which this structure is coded up) has a "looser" metric structure. (I explore this difference in North (20I9).) Hamiltonian mechanics allows for even

\footnotetext{
${ }^{21}$ Lanczos notes that the Lagrangian equations "stand out as the first example of that "principle of invariance' [a kind of coordinate-independence] which was one of the leading ideas of I $9^{\text {th }}$ century mathematics, and which has become of dominant importance in contemporary physics" (1970, I I 7 ).

${ }^{22}$ Einstein said that the main reason it took him so long to develop general relativity is that "it is not so easy to free oneself from the idea that co-oordinates must have an immediate metrical meaning" (Schilpp, I970, 67).
} 
greater freedom of coordinates than that. (In particular, it allows for coordinate changes that mix up the $p$ 's and $q$ 's, whereas in Lagrangian mechanics, since $\dot{q}$ is defined as the time derivative of $q$, there is no allowable transformation in which these "get intermingled" (Taylor, 2005, 538). ${ }^{23}$ ) The result is that the Hamiltonian formulation does not even require a metric structure, but only a lesser type of structure akin to a volume measure. (I explore this difference in North (2009).)

I'd go so far as to suggest that there is a hierarchy, in order of increasing mathematical structure, from Hamiltonian to Lagrangian to Newtonian mechanics-a mathematical nonequivalence among the three. (In the above-mentioned writings, I argue that less such structure is in general a reason to prefer a theory.) If we take a theory's mathematical structure seriously in telling us about the nature of the physical world, then this mathematical difference should reflect a similar hierarchy in the physical structure of the world each theory describes-a physical nonequivalence among them. In other words, these may not be wholly equivalent formulations, neither mathematically nor physically, contrary to the standard view. ${ }^{24}$

\subsection{Metaphysical differences}

Since the dynamical equations are inter-derivable in the way that physics books claim, you might conclude that the three formulations are simply "mutually supporting, compatible perspectives on the phenomena of mechanical motions" (Wilson, 2007, I79). That is the standard view. ${ }^{25}$ But there are other differences among the formulations, what I call here "metaphysical" ones, that could lead to a different conclusion. Although no theory wears its metaphysics on its sleeves, on some natural interpretations they differ from one another in potentially significant ways. All assume a fundamental ontology of point-mass particles with relative positions, ${ }^{26}$ but beyond that each one offers a fairly different fundamental picture of the world, given the different quantities that appear in their respective

${ }^{23}$ There is a mathematical transformation between them (note I9), but even it "leads one to suspect that there actually is a nontrivial difference between $L$ and $\dot{q}$ on the one hand and $H$ and $p$ on the other" (José and Saletan, 1998, 2 I 7 ).

${ }^{24}$ Opposition to the above in philosophy is in Swanson and Halvorson (2OI 2); Curiel (2OI4); Barrett (20I5). Barrett (20I8) points out how our judgments about the relationship between the theories will depend on what we take to be the theories' core structures, with different views on the latter leading to different judgments about the former.

${ }^{25}$ Following Coffey (2014), the standard view might more accurately be put as that Newtonian mechanics accurately represents physical reality, with Lagrangian and Hamiltonian mechanics being reformulations of it.

${ }^{26}$ Though see note 5 . 
dynamical equations. (What follows are some initial suggestions; the metaphysics of the three formulations has not been much explored in the literature.)

First compare Newtonian mechanics, on the one hand, with Lagrangian and Hamiltonian mechanics, on the other. Newtonian mechanics "describes the world in terms of forces and accelerations (as related by the second law)" (Taylor, 2005, 52 I), where "force is something primitive and irreducible" (Lanczos, I970, 27). Lagrangian and Hamiltonian mechanics describe systems in terms of energy, with force being "a secondary quantity" derivable from the energy (Lanczos, I970, 27). According to Newtonian mechanics, the world is fundamentally made up of particles that move around in response to various forces between them. According to Lagrangian and Hamiltonian mechanics, particles move around and interact as a result of their energies. Although energy and force functions are inter-derivable in ways that physics books will show (albeit under certain contestable assumptions: note 9), these are nonetheless prima facie different pictures of the world, built up out of different fundamental quantities, with correspondingly different explanations of the phenomena. Compare: the Schrödinger and Heisenberg formulations of quantum mechanics are generally considered inter-derivable, yet you might not want to regard them as wholly metaphysically equivalent even so; many philosophers take only the former to directly or perspicuously represent what is going on physically, for instance. (You might think that Lagrangian and Hamiltonian mechanics can be seen as fundamentally forced-based, given in terms of "generalized forces." However, generalized forces are so-called by analogy to ordinary forces. It isn't clear that they count as regular forces of the Newtonian kind.)

There are potential metaphysical differences between the two energy-based approaches as well. In Lagrangian mechanics, generalized velocities are defined as the first time derivatives of the generalized positions. This suggests that (generalized) positions are the only truly fundamental dynamical features of the particles, the velocities being defined in terms of them. In Hamiltonian mechanics, on the other hand, the canonical positions and momenta are both independent variables, neither being defined in terms of the other: both appear to be fundamental. (This, in turn, may amount to an "impetus" view in the medieval tradition, with further metaphysical repercussions: Arntzenius (2000). This assumes that the second law of Hamiltonian mechanics is not a definition of the generalized momentum, as often claimed, but a further fundamental dynamical law.) Another difference is that the Hamiltonian is typically equal to the total energy of a system, whereas the Lagrangian is the difference between the kinetic and potential energies. Perhaps this, too, amounts to a real difference. ${ }^{27}$

${ }^{27} \mathrm{Baez}(2005$, ch. I) tries to distinguish them physically. 
In fact, there is a range of potential views on what's fundamental to each of the formulations, and it is not clear which is correct. It is an open question whether, on any of them, ordinary three-dimensional space is the fundamental space, or whether what we usually think of as the merely abstract, high-dimensional statespace (or the configuration space) is. Relatedly, it is open whether particle features like positions and momenta are fundamentally defined on the lowor high-dimensional space. (Compare the debate in quantum mechanics over the fundamentality of the high-dimensional space of the wavefunction versus ordinary three-dimensional space.) Within energy-based approaches, it is open whether the energy function, $L$ or $H$, is fundamental, or whether instead the potential and kinetic energies are; or indeed whether any energy quantity is fundamental, rather than the particle positions and velocities in terms of which the energy is standardly defined; or whether all of these might be fundamental. Analogous questions arise for Newtonian mechanics: are total forces or component forces fundamental? ${ }^{28}$ For that matter, can Newtonian mechanics be seen as a fundamentally energy-based theory, given the inter-derivability of the different quantities? ${ }^{29}$ Finally: are any of these genuinely distinct possibilities, or are they all equivalent, just different, equally legitimate ways of describing the same physical reality, analogous to the different coordinate-based descriptions of the plane? Although physics books generally assume the latter, notice that certain metaphysical views will say that only one description gets at the real or fundamental properties (Lewis, I983; Sider, 201 I).

In all, it seems very much an open question whether the three main formulations of classical mechanics are genuinely equivalent, just notational variants of a single theory, as usually thought. There is a case to be made that the differences are significant enough to render them more like distinct theories, with different accounts of what the physical world is like. All of this warrants further investigation.

\section{References}

Allori, Valia (20 I 7). "To 'Mass', or Not to 'Mass', that is the 'Matter': Laws of Nature, Fundamental Properties, and Ontology." Unpublished manuscript.

Arnold, V. I. (1989). Mathematical Methods of Classical Mechanics. New York: Springer-Verlag, 2nd edn. Translated by K. Vogtmann and A. Weinstein. First edition 1978 .

\footnotetext{
${ }^{28}$ Cartwright (1983, ch. 3) argues against the reality of component forces.

${ }^{29}$ Wilson (2007) defends the existence of Newtonian forces against various objections.
} 
Arntzenius, Frank (2000). "Are There Really Instantaneous Velocities?" The Monist 83, I 87-208.

Baez, John (2005). "Lectures on Classical Mechanics." URL http://math.ucr.edu/home/baez/classical/texfiles/2005/ book/classical.pdf.

Barrett, Thomas (2018). "Equivalent and Inequivalent Formulations of Classical Mechanics." British Fournal for the Philosophy of Science https: / / do i . org/ 10.1093/bjps/axy017.

Barrett, Thomas William (2015). "On the Structure of Classical Mechanics." British fournal for the Philosophy of Science 66, 80 I-828.

Brading, Katherine and Elena Castellani (2007). "Symmetries and Invariances in Classical Physics." In Jeremy Butterfield and John Earman, eds., Handbook of the Philosophy of Science: Philosophy of Physics, Part B, pp. I33 I-I 367 . Amsterdam: Elsevier.

Butterfield, Jeremy (2004). "Between Laws and Models: Some Philosophical Morals of Lagrangian Mechanics." Available at http:// philsci-archive.pitt.edu/1937/.

Callender, Craig (1995). "The Metaphysics of Time Reversal: Hutchison on Classical Mechanics." British Fournal for the Philosophy of Science 46, 33 I-340.

Cartwright, Nancy (1983). How the Laws of Physics Lie. Oxford: Oxford University Press.

Coffey, Kevin (2014). "Theoretical Equivalence as Interpretive Equivalence." British fournal for the Philosophy of Science 65, 82 I-844.

Curiel, Erik (2OI4). "Classical Mechanics Is Lagrangian; It Is Not Hamiltonian." British Fournal for the Philosophy of Science 65, 269-32 I.

Earman, John (1986). A Primer on Determinism, vol. 32. University of Western Ontario Series in the Philosophy of Science: Reidel.

Esfeld, Michael, Andrea Oldofredi Dirk-André Deckert with Dustin Lazarovici, and Antonio Vassallo (20 18). What There Is: A Minimalist Ontology of the Natural World. New York and London: Routledge.

Feynman, Richard (1965). The Character of Physical Law. Cambridge, Mass.: MIT Press. 
Feynman, Richard P., Robert B. Leighton, and Matthew Sands (2006). The Feynman Lectures on Physics: The Definitive Edition, vol. I. Reading, Mass.: Addison-Wesley. First edition published in 1964.

Gallavotti, Giovanni (1983). The Elements of Mechanics. New York: SpringerVerlag.

Goldstein, Herbert, Charles Poole, and John Safko (2004). Classical Mechanics (Third Edition). Reading, Mass.: Pearson Education. First edition published in I950.

Hall, Ned (2007). "Humean Reductionism about Laws of Nature." Unpublished manuscript. Available at http://philpapers.org/archive/HALHRA.

Hand, Louis N. and Janet D. Finch (1998). Analytical Mechanics. New York: Cambridge University.

Hertz, Heinrich (1899). The Principles of Mechanics Presented in a New Form. London: Macmillan and Co.

Hicks, Michael Townsen and Jonathan Schaffer (201 7). "Derivative Properties in Fundamental Laws." The British fournal for the Philosophy of Science 68(2), 4II -45 O.

Hutchison, Keith (1993). "Is Classical Mechanics Really Time-Reversible and Deterministic?" British Fournal for the Philosophy of Science 44, 307-323.

José, Jorge V. and Eugene J. Saletan (I998). Classical Dynamics: A Contemporary Approach. Cambridge: Cambridge University.

Lanczos, Cornelius (1970). The Variational Principles of Mechanics. New York: Dover, $4^{\text {th }}$ edn. First edition published in 1949 .

Lewis, David (1983). "New Work for a Theory of Universals." Australasian Fournal of Philosophy 61, 343-77.

Malament, David (2008). “Norton's Slippery Slope.” Philosophy of Science (Proceedings) 75(5), 799-8 16 .

Marion, Jerry B. and Stephen T. Thornton (1995). Classical Dynamics of Particles and Systems. Florida: Harcourt, Brace and Company, 4th edn.

North, Jill (2009). "The 'Structure' of Physics: A Case Study." Fournal of Philosophy 106(2), 57-88. 
- (2019). Structure, Physics, and Reality. Unpublished manuscript.

Norton, John (2008). “The Dome: An Unexpectedly Simple Failure of Determinism." Philosophy of Science (Proceedings) 75(5), 786-798.

Schilpp, Paul Arthur, ed. (1970). Albert Einstein: Philosopher-Scientist. La Salle, IL: Open Court, 3 rd edn. First edition published I947.

Sider, Theodore (20 I I). Writing the Book of the World. Oxford and New York: Oxford University Press.

Sklar, Lawrence (2013). Philosophy and the Foundations of Dynamics. Cambridge: Cambridge University Press.

Spivak, Michael (20I0). Physics for Mathematicians: Mechanics I. United States: Publish or Perish.

Sussman, Gerald Jay and Jack Wisdom (2014). Structure and Interpretation of Classical Mechanics. Cambridge, Mass.: MIT Press, 2nd edn.

Swanson, Noel and Hans Halvorson (2012). "On North's 'The Structure of Physics'." Available at http://philsci-archive.pitt.edu/9314/.

Talman, Richard (2000). Geometric Mechanics. New York: John Wiley \& Sons.

Taylor, John R. (2005). Classical Mechanics. Sausalito, California: University Science Books.

Wilson, Jessica (2007). "Newtonian Forces." British fournal for the Philosophy of Science 58, I 73-205.

Wilson, Mark (2009). "Determinism and the Mystery of the Missing Physics." British fournal for the Philosophy of Science 60, I 73-193.

_ (2013). "What is 'Classical Mechanics' Anyway?" In Robert Batterman, ed., The Oxford Handbook of Philosophy of Physics, pp. 43-106. Oxford: Oxford University.

_ (forthcoming). "Newton and the Billiard Ball." In Chris Smeenk and Eric Schliesser, eds., The Oxford Handbook of Newton. Oxford University. 